\title{
Pain management in weight loss surgery: aiming for multimodal approach
}

\begin{abstract}
Weight loss surgery (WLS) is an effective group of procedures with a low rate of complications, preventing or treating several cardiovascular co morbidities associated with morbid obesity. Pain management in the preoperative needs special consideration in this population because of different aspects, specially the theoretical need for opioid avoidance, generally used in the postoperative of major surgeries. This literature review assesses the available information on analgesic strategies for WLS, with a special approach on multimodal settings and possible alternatives. Well-controlled studies are needed to determine the level of recommendation for individual therapies and multimodal regimens.
\end{abstract}

Keywords: weight loss surgery, bariatric surgery, obesity, pain management, postoperative, pain
Volume 5 Issue 2 - 2016

\author{
Juan S Barajas Gamboa,' Daniel Gonzalez \\ Nuñez ${ }^{2}$ \\ 'Department of Surgery, Autonomous University of \\ Bucaramanga, Colombia \\ 2Department of Surgery, Pontifical Xavierian University, \\ Colombia
}

Correspondence: Juan S Barajas Gamboa MD, Department of Surgery, Autonomous University of Bucaramanga, Floridablanca, Santander, Colombia, Email jbarajasgamboa@gmail.com

Received: September 26, 2016 | Published: November 04

\section{Introduction}

The importance of WLS can be explained by the epidemic of obesity not only in the United States, where rates according to the Center for Disease Control (CDC) are between 26 and 32\% (and rising). It is expected that almost half of the Americans will be overweight by 2018. Surgical approaches still remain the preferred alternative for this public health problem. Most of the bariatric surgery procedures are performed using minimally invasive techniques (MIS), these include laparoscopic gastric bypass, Laparoscopic Sleeve Gastrectomy, Laparoscopic Gastric Banding and Laparoscopic Biliopancreatic Diversion with Duodenal Switch (Figure 1) (Table 1).

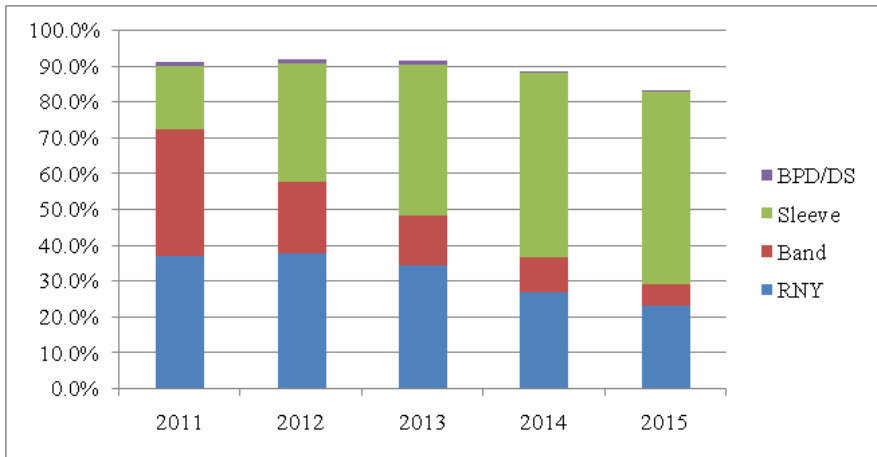

Figure I Minimally invasive bariatric surgery statistics.

Table I BOLD,ASC/MBSAQIP, National inpatient Sample data and outpatient estimations

\begin{tabular}{llllll}
\hline & $\mathbf{2 0 I 1}$ & $\mathbf{2 0 1 2}$ & $\mathbf{2 0 1 3}$ & $\mathbf{2 0 1 4}$ & $\mathbf{2 0 1 5}$ \\
\hline Total (thousands) & 158 & 173 & 179 & 193 & 196 \\
RNY & $36.70 \%$ & $37.50 \%$ & $34.20 \%$ & $26.80 \%$ & $23.10 \%$ \\
Band & $35.40 \%$ & $20.20 \%$ & $14 \%$ & $9.50 \%$ & $5.70 \%$ \\
Sleeve & $17.80 \%$ & $33 \%$ & $42.10 \%$ & $51.70 \%$ & $53.80 \%$ \\
BPD/DS & $0.90 \%$ & $1 \%$ & $1 \%$ & $0.40 \%$ & $0.60 \%$ \\
\hline
\end{tabular}

One of the clinical advantages of using MIS approaches is less probability of postoperative pain. The estimated annual medical cost of obesity in the U.S. in 2008 was \$147billion U.S. dollars. The average mortality rate associated with bariatric surgery was just $0.13 \%$.

Pain management strategies are extensive and diverse. From physical modalities to intravenous infusion of new sedoanalgesic agents, therapeutic alternatives are at hand. The key to reaching good clinical practices on preoperative pain control is to individualize procedures, populations and patients. It is widely accepted the different perception of pain that patients experience in similar conditions, therefore the need to guide its management with objective scales and effective agents.

Bariatric surgery presents a difficult population due to a high prevalence of associated OSA (Obstructive Sleep Apnea) and cardiovascular risk factors with a theoretical need for less opioid and NSAID consumption, among other elements. A study evaluating obese patients programmed for WLS found an average of $78 \%$ of OSA in a formal sleep study, clearly rising according to BMI. ${ }^{1}$ This high incidence is translated into preoperative hypoxia; an increased risk of postoperative complications, higher need of ICU and increased hospital stay. ${ }^{2}$ Opioids have been shown to impair ventilatory function by affecting both peripheral and central carbon dioxide chemo reflex loops,$^{3}$ having special contraindication in patients at high-risk of preoperative hypoxia such as OSA.

Another aspect to be taken into account regarding opioid use is the associated bowel dysfunction. With varying prevalence in different studies it is an important cause of postoperative morbidity and increased hospital stay. Most commonly presenting as constipation it can also be accounted for decreased gastric emptying, abdominal pain, bloating and delayed gastrointestinal transit. ${ }^{4}$

As important as multimodal management of pain is, the effectiveness of each strategy must be evaluated in light of high quality evidence. We present the assessment of strategies used in bariatric surgery with a discussion regarding the clinical implications and possible protocols to be used in the preoperative, especially in 
post-anesthetic care units (PACU) and surgical wards. The aim is to reduce postoperative pain while achieving an adequate safety profile in obese patients.

\section{Methods}

A medical literature review was conducted in order to find the most recent medical manuscripts published in this clinical field. Using the Keywords: Weight Loss Surgery, Bariatric Surgery, Obesity, Pain Management, Postoperative and Pain the following databases were consulted: Pubmed, Lilacs, Proquest, Cochrane, Ovid and Google Scholar. With the aim to maximize the number of articles, no date limit in the search was set, and manuscripts in English, Spanish and Portuguese were considered. Meta-analysis papers, review articles, clinical trials, prospective and retrospective case series were included in the review.

Initially, 314 articles were obtained, of which 152 met the inclusion criteria. However, only the 34 were referenced in the current work with a focus on bariatric surgery and pain management. Considering the nature of this publication, no ethical approval and statistical consultation were needed in its preparation.

\section{Review}

\section{Pain assessment}

The World Health Organization defines pain as "an unpleasant sensory or emotional experience associated with actual or potential tissue damage, or described in terms of such damage". The difficulty to objectively assess pain in patients has demanded the use of scales and questionnaires to identify classify and ultimately manage pain. Validated scales are accurate because of tested intrapatient and inter-rater reliability. Amongst these, the Visual Analogue Scale (VAS) (Figure 2), Faces Rating Scale (Figure 3) and International Pain Outcomes Questionnaire are frequently used in post operatory pain rating studies. It is important to not only determine severity but to differentiate its characteristics, effect on life quality and early mobilization as well as response to pharmacological and other pain relieving modalities. ${ }^{5}$

\section{Visual Analog Scale (VAS)*}

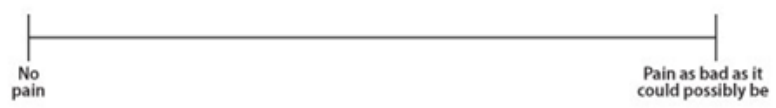

Figure 2 Visual analog scale (VAS).

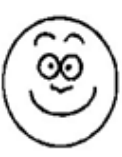

0

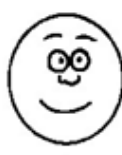

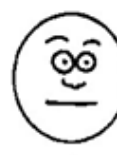

4
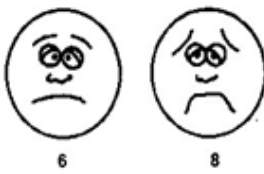

8

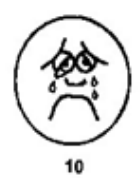

10
Figure 3 Faces rating scale.

\section{Therapeutic alternatives}

\section{Premedication}

The only study found to date evaluating the effect on premedication in bariatric surgery is the administration of $300 \mathrm{mg}$ of Pregabalin in the preparatory period. Outcomes on pain intensity levels were consistently lower and nausea/vomiting decreased in the study group. No opioid use or effect on mobilization was assessed (Table 2). ${ }^{6}$

Table 2 Local anesthesia Vs. regional anesthesia approaches and clinical outcomes

\begin{tabular}{lll}
\hline & $\begin{array}{l}\text { Median pain } \\
\text { score at 24h }\end{array}$ & $\begin{array}{l}\text { Need for morphine } \\
\text { rescue**** }\end{array}$ \\
\hline IV analgesia* & 5 & $16 \%$ \\
IV analgesia + Epidural** & 2.5 & 2 \\
$\begin{array}{l}\text { IV analgesia + Local } \\
\text { anesthetic*** }\end{array}$ & 2 & 2 \\
\hline
\end{tabular}

*Metamizole $2 \mathrm{~g} / 8 \mathrm{~h}$ and acetaminophen $\mathrm{Ig} / 8 \mathrm{~h}$, alternating every $4 \mathrm{~h}$.

*** Continuous perfusion of levobupivacaine $0.125 \%$

$* * * 10 \mathrm{ml}$ of bupivacaine $0.25 \%$, applying $2 \mathrm{ml}$ under the aponeurotic layer in each port.

$* * * * 5 \mathrm{mg}$ of subcutaneous morphine.

\section{Transverses abdominis plane block (TAP) block}

The use of TAP block technique has been rising since its description in 2001. Designed to provide anesthesia in the anterior abdominal wall (T6-L1) it has demonstrated a reduction in pain scores and opioid consumption in early post operatory for abdominal and gynecological surgery. ${ }^{7}$

Studies performed in bariatric surgery include a single-port laparoscopy for sleeve gastrectomy cohort study in which pain scores $12-24 \mathrm{~h}$ post surgery were significantly better but no differences in opioid consumption were achieved, ${ }^{8}$ nonetheless a recent Randomized Clinical Trial (RCT) concluded that TAP blocks do not provide additional analgesic benefit when added to trocar insertion site anesthetic infiltration with systemic analgesia for laparoscopic gastric-bypass surgery. ${ }^{9}$

Because of the difficulty of anatomical references in obese patients, ultrasound guided (USG) TAP blocks have been reevaluated. ${ }^{10}$ Sinha et al., ${ }^{11}$ demonstrated improvement of three different variables: lower pain scores in VAS, less need for opioids (Tramadol) therefore allowing for less sedation and early ambulation. Patient satisfaction scores were also significantly higher. ${ }^{11}$

\section{Local anesthetics: port site infiltration}

A prospective randomized study of patients undergoing laparoscopic sleeve gastrectomy compared three analgesic strategies finding no differences in operation time, complications, mortality or hospital stay. ${ }^{12}$ Strategies and positive clinical findings in pain reduction and opioid use are summarized in Table 3. A different finding was achieved in a retrospective randomized study where VAS scores were lower in the study group (bupivacaine infiltration) in the first 4h postoperatively without reaching statistical significance and did not differ in any other period of time. Also, no statistically significant differences in pain perception or the need for rescue medication were evidenced. ${ }^{13}$

\section{Local anesthetics: intraperitoneally - subfascially/ subcutaneously}

Two different studies evaluated intraperitoneal infiltration 
of bupivacaine with improved pain scores within the first $24 \mathrm{~h}$ postoperatively and reduction of postoperative hydrocodone/ acetaminophen requirements compared with the placebo group although study quality was poor and both results lacked clinical relevance. ${ }^{14,15}$ Postoperative subfascial/subcutaneous infusion of bupivacaine had no effect compared to placebo on pain, opioid use or hospital length stay. ${ }^{16}$

Table 3 Multimodal Analgesia protocol Lam et al. ${ }^{22}$

\begin{tabular}{|c|c|}
\hline \multicolumn{2}{|l|}{ Analgesia Exclusively } \\
\hline Stage & Protocol \\
\hline \multirow{3}{*}{ Premedication } & Acetaminophen $2 \mathrm{~g}$ \\
\hline & Pregabalin 300/I50mg (BMI adjusted) \\
\hline & Dexmetomedine $2 \mathrm{ug} / \mathrm{kg} / \mathrm{h}^{*}$ \\
\hline Induction & Fentanyl I00ug \\
\hline Maintenance & Dexmetomedine $>0.2 \mathrm{ug} / \mathrm{kg} / \mathrm{h}$ \\
\hline \multirow{4}{*}{ Intraoperative analgesia } & $\begin{array}{l}\text { Ketamine bolus } 0.3 \mathrm{mg} / \mathrm{kg} \text { (intermittent } \\
0.2 \mathrm{mg} / \mathrm{kg} / \mathrm{h} \text { afterwards) }\end{array}$ \\
\hline & Parecoxib 40mg \\
\hline & Tramadol 100mg \\
\hline & Local anesthetic infiltration** \\
\hline Rescue analgesia in PACU & $\begin{array}{l}\text { Pethidine iv } 20 \mathrm{mg} \text { with Ketamine iv } 5 \mathrm{mg} \text { if } \\
\text { necessary }\end{array}$ \\
\hline \multirow{2}{*}{ Routine analgesia in ward } & Etoricoxib $120 \mathrm{mg}$ po daily \\
\hline & Pregabalin $75 \mathrm{mg}$ at night \\
\hline Rescue analgesia in ward & $\begin{array}{l}\text { Pethidine im } 50 \mathrm{mg} \text { every } 4 \text { hours as } \\
\text { required }\end{array}$ \\
\hline
\end{tabular}

*Using LBW (lean body weight)

**Levopubivacaine $0.5 \% 20 \mathrm{ml}$.

Dexmetomedine: The use of Dexmetomedine (DXM) has been studied in two recent papers due to its safety profile regarding respiratory depression and better hemodynamic stability. Intraoperative fentanyl and propofol were reduced as well as pain scores and postoperative use of PCA (patient-controlled analgesia). ${ }^{17}$ Another study demonstrated reduced desflurane and rescue fentanyl/antiemetic requirement in the PACU with lower arterial blood pressure measurements. ${ }^{18}$

NSAIDs: A non placebo-controlled study comparing Ketorolac and Remifentanyl infusion during surgery found lower requirements of PCA, nausea/vomiting, duration of stay in PACU and improved pain scores. ${ }^{19}$

Ketamine: The placebo-controlled study against ketamine administration showed intraoperative reduction of Remifentanyl and Propofol requirements as well as improved pain scores and less use of PCA morphine in the PACU. ${ }^{20}$

Acetaminophen: A study evaluating the effectiveness of IV Acetaminophen in perioperative opioid reduction found lower cumulative 24-hour opioid use with mean reductions of morphine equivalents being $21.1 \mathrm{mg}$ for the LSG group, $32.6 \mathrm{mg}$ for the LRYGB group, and $27.7 \mathrm{mg}$ for the combined group. No differences in mean pain scores were achieved. ${ }^{21}$

\section{Multimodal analgesia in bariatric surgery}

The combination of different analgesic therapies is not a new concept. Its purpose is to target more than one pain mechanism, overall diminishing pain perception and providing a reduction in dosages and adverse effects.

Taking into account the importance of multimodal analgesia in the perioperative period, bariatric surgery has several issues that demand this approach. With a wide variety of alternatives available in the intraoperative, the PACU and the surgical ward some pharmacological therapies are contraindicated or have serious adverse effect in obese patients. Although there are no controlled trials comparing multimodal approaches, bariatric surgery departments are creating protocols aiming to diminish opioid and NSAID use.

In a prospective observational study, Lam et al. used a multimodal analgesia regimen with a combination of acetaminophen, pregabalin, COX-2 inhibitors, tramadol, ketamine, dexmedetomidine, and local anaesthetic wound infiltration achieving no opioid use in up to $46 \%$ and rescue opioids exclusively in the post-anesthetic care unit in another $20 \%$. Table 4 summarizes their analgesia regimen. ${ }^{22}$

Table 4 Suggested therapeutic approaches

\begin{tabular}{lllll}
\hline & Preoperatory & Intraoperative & PACU & Surgical ward \\
\hline \multirow{3}{*}{ Strong Recommendation } & Acetaminophen & Tramadol & NPCOIA** & Pregabalin \\
& TAP* Block & NSAID & Acetaminophen & NPCOIA \\
& NSAID** & Local anesthetic/Epidural & NSAID & Acetaminophen \\
& Pregabalin & Dexamethasone & & NSAID \\
Moderate Recommendation & DXM***** & DXM & PCOIA*** & PCOIA \\
& & Ketamine & Ketamine & Other modalities \\
Weak Recommendation & COX-2**** & COX-2 & Scheduled Opioids & COX-2
\end{tabular}

* Transversus Abdominis Plane

**Non-patient controlled opioid IV analgesia

****Patient-controlled Opioid IV analgesia

****Cyclooxygenase-2 inhibitor

******Dexmetomedine

Citation: Barajas Gamboa MD, Gonzalez Nuñez MD. Pain management in weight loss surgery: aiming for multimodal approach. Adv Obes Weight Manag Control. 2016;5(2):232-237. DOI: 10.15406/aowmc.2016.05.00I25 


\section{Discussion}

As described by several authors, obese patients have special characteristics that lead to a different approach in anesthetic and analgesic management. Physiological aspects include OSA, a main risk factor in preoperative hypoxia, different biodistribution of medical compounds and cardiovascular impairments. The use of Lean Body Weight LBW appears to be a more accurate way to determine dosage and prescription by excluding body fat from the total body weight equation. ${ }^{5,22}$

Research on adequate postoperative pain management is a current trend in post-anesthetic and surgical areas. Objective assessment and patient satisfaction degree are parameters of quality medical attention. Duration of high pain scores increase the incidence of post-operative morbidity and lead to delayed recovery, prolonged hospital stay and increased healthcare costs.

After a complete and thorough medical literature review we found a lack of high quality evidence studies to support individual and combined therapies. No multicenter randomized clinical trials and consensuses are focused in pain management in the bariatric surgery field, only observational and single-site case series suggest and propose therapeutic approaches.

Veerabhadram has described a general overview of actual postoperative pain management. The basis of it being to eliminate pain with as little side effects as possible, taking into account special populations and procedure specifics. The different modalities (patient controlled/“as needed"), routes (oral, IV, regional and neuraxial) and agents (opioid/non-opioid) used can determine a modifiable regimen. Their approach includes preoperative Acetaminophen and Ketorolac, intraoperative wound infiltration, and postoperative Acetaminophen, Ibuprofen, PCIA and Oxycodone. ${ }^{23}$

In a prospective observational study published in 2013 by Dr. Aliya Ahmed et al., ${ }^{24}$ authors outlined pain management strategies used after major abdominal surgeries in a tertiary care hospital. Patients undergoing major abdominal surgery were included in the study and clinical outcomes such as postoperative analgesic strategies, analgesics used, pain and sedation scores, collateral symptoms were evaluated, ultimately analyzing patient satisfaction. Over 100 patients enrolled the clinical study showing that epidural analgesia was used in $61 \%$; PCIA was found in $21 \%$ and opioid infusion in around 14 patients. Multimodal analgesia was employed in 98 patients. 99 patients reported a satisfactory experience with the analgesic alternative. ${ }^{24}$

Vallano et al. ${ }^{25}$ described the patterns of prescription and administration of analgesic drugs used for the postoperative pain after abdominal surgery in Spanish hospitals. Authors planned to know the prevalence and severity of postoperative pains amongst the participating centers. The study was designed as a descriptive cross-sectional multicenter drug utilization study in twelve reference hospitals in Spain. 993 patients were included in this trial and the most common surgeries performed were inguinal hernia repair, cholecystectomy, appendectomy, bowel resection and gastric surgery. $55 \%$ of patients only received non-opioid analgesics, $9 \%$ received only opioid analgesics and $27 \%$ received both. The most common analgesics used were Metamizol and Pethidine. ${ }^{25}$

These studies give a general overview of commonly used pain management strategies in abdominal surgeries, being for the greater part a combination of routes, agents and dosages. Their importance relies on the demonstration that multimodal analgesia is a not a concept, it is an ongoing strategy with multiple variables, yet to be specified and instituted as a guideline.

The multimodal protocol proposed by Lam et al has several limitations if used as a guideline. There is no way to assess the effectiveness of single therapy versus multimodal regimen or differences with other protocols. Also, rescue opioid was administered differently and intramuscular dosage route can have an erratic absorption. The individual efficacy of each therapy can be discussed in light of other studies.

In recent years a number of physical therapies have been proposed for postoperative pain relief, nevertheless the only modality with sufficient evidence for recommendation is transcutaneous electrical nerve stimulation (TENS) as adjunctive to other approaches, with no specific studies performed in WLS. ${ }^{26}$

Gabapentinoids are gaining importance in multimodal approach. In a recent meta-analysis by Zhang et al including mainly abdominal and gynecological trials, Pregabalin demonstrated a dose-related nausea/ vomiting and opioid use reduction but increased visual disturbances. ${ }^{27}$ With few adverse effects and promising results this pharmacological group can be added to ongoing protocols.

COX-2 inhibitors have to be used with cautiousness because cardiovascular mortality is a major issue in patients undergoing bariatric surgery, often presenting various risk factors. In 2013 the FDA (US Food and Drug Administration) issued a warning regarding their use due to increased CV mortality, life-threatening skin reactions and lack of superior efficacy compared to other NSAIDs. Studies in colorectal procedures and animal models have correlated NSAIDS with anastomotic leakage, ${ }^{28}$ representing a potential risk in bariatric surgery, not studied up to date. Positive findings with Ketorolac use are not exempt of the same risks and adverse effects, addressing potentially serious gastrointestinal bleeding. Despite these associations, there appears to be no additional harm regarding NSAIDS in a short (perioperative) course.

Infiltration of incision sites in laparoscopy has shown low quality evidence with conflicting findings in a series of studies, hence the latest pain management guidelines recommendation to decide based on procedure-specific results. ${ }^{5}$ In a randomized, controlled study in laparoscopic cholecystectomy, incision infiltration proved to be better at reducing postoperative pain than in the intraperitoneal group. ${ }^{29}$ USguided TAP block is a promising approach but well-controlled clinical trials need to evaluate its efficacy compared to placebo, epidural and local (port-site, intraperitoneal) infiltration.

Historically, the World Health Organization proposed an ascending ladder guiding pain management therapy for both acute and chronic pain. Opioids were divided into strong and weak opioids based on a theoretical better safety profile of the latter but there is no evidence supporting this assumption. At equivalent analgesic effect, weak opioids such as tramadol have no additional differences compared to NSAIDs and can have the serious adverse effects usually attributed to strong opioids. Tramadol is frequently used in the intraoperative and postoperative setting as part of multimodal approach, in the absence of strong evidence against its use it is a valid alternative.

Schnabel et al found intraoperative DXM leads to lower postoperative pain, reduced opioid consumption, and a lower risk for opioid-related adverse events. In bariatric surgery, studies evaluating its effectiveness failed to perform control groups with specific analgesia strategies and clinical relevance of findings appears to be minimal. Some adverse effects such as hypotension and bradycardia require caution in patients at risk. ${ }^{30}$ 
In May 2016, a meta-analysis from Cochrane including 7200 patients from 75 studies determined that with a single $500-1000 \mathrm{mg}$ postoperative dose of IV Acetaminophen, 36\% of patients could have a $50 \%$ pain relief over 4-6hours compared to placebo. Opioid use was also reduced, although no change in opioid-induced adverse events was observed. ${ }^{31}$ Preoperative acetaminophen in abdominal hysterectomy was able to reduce opioid consumption and related adverse effects without a significant reduction in pain scores. ${ }^{32}$

A meta-analysis conducted in 2011 included 24 randomized trials in perioperative Dexamethasone, having as primary outcome pain scores (acute/late) and $24 \mathrm{~h}$ opioid consumption. Their conclusions were that an intermediate dose of $0.11-0.2 \mathrm{mg} / \mathrm{kg}$ (more clearly with preoperative administration) had opioid-sparing effects, less nausea/ vomiting and lower pain scores. ${ }^{33}$

In a recent updated Cochrane review on patient controlled opioid analgesia versus non-patient controlled opioid analgesia in the treatment of postoperative pain author's evaluated efficacy and safety as primary outcomes. Patients often preferred PCA and pain scores were slightly lower in this group, this can be attributed to a minor increase in opioid doses. Side effects were similar apart from higher pruritus presented in PCA group. ${ }^{34}$

Based on previous studies and analyses performed by experts in the field we suggest a multimodal approach with specific recommendations grouped by levels of efficacy, safety and availability (Table 4). Our intention is merely to encourage the creation of protocols in each institution by the medical team including anesthesia, surgery, nurse staff and adjoined departments.

One of the most important limitations of this manuscript (literature review) is reliance on previously published studies and their proven appropriate methods and methodologies.

In the near future, multidisciplinary teams should be able to gradually introduce protocols in pain management of the obese patient in the preoperative as individual and multimodal regimens are supported by evidence. The aim is to reduce or eliminate the need for opiates therefore having less respiratory depression, nausea/vomiting and postoperative ileus. On the other hand, it is expected that researchers in the pharmaceutical industry, develop new generations of analgesics with better safety profile and higher efficacy.

\section{Conclusion}

WLs is a growing field in medicine; as surgical approaches become less invasive with new techniques, surgeons are able to lower complications and mortality to a minimum rate. Pain is rarely viewed as a complication thus its poor management in a wide proportion of postoperative patients. Obese patients require a special approach mainly because of cardiovascular risk factors, obesity itself and OSA, limiting both pharmacological and non-pharmacological treatments.

The need for opioid and pain experience reduction in the preoperative has encouraged the study and use of other alternatives. Specific, head-to-head studies and critical analyses of available data in bariatric surgery are yet to be conducted regarding multimodal analgesia and single therapeutic alternatives. There are institutional protocols based on preliminary encouraging results that have reduced main variables (pain scores, opioid use, mobilization and hospital stay), however randomized clinical trials are still needed to clarify preliminary data and establish international guidelines with strong evidence.

\section{Acknowledgements}

None.

\section{Conflict of interest}

The author declares no conflict of interest.

\section{References}

1. Lopez PP, Stefan B, Schulman CI, et al. Prevalence of sleep apnea in morbidly obese patients who presented for weight loss surgery evaluation:more evidence for routine screening for obstructive sleep apnea before weight loss surgery. Am Surg. 2008;74(9):834-838.

2. Gupta RM, Parvizi J, Hanssen AD, et al. Postoperative complications in patients with obstructive sleep apnea syndrome undergoing hip or knee replacement:a case-control study. Mayo Clin Proc. 2001;76(9):897-905.

3. Borison HL. Central nervous respiratory depressants-narcotic analgesics. Pharmacol Ther B. 1977;3(2):227-237.

4. Panchal SJ, Müller-Schwefe P, Wurzelmann JI. Opioid-induced bowel dysfunction: prevalence, pathophysiology and burden. International Journal of Clinical Practice. 2007;61(7):1181-1187.

5. Chou R, Gordon DB, de Leon-Casasola O, et al. Management of Postoperative Pain: A Clinical Practice Guideline from the American Pain Society, the American Society of Regional Anaesthesia and Pain Medicine, and the American Society of Anesthesiologists' Committee on Regional Anaesthesia, Executive Committee, and Administrative Council. J Pain. 2016;17(2):131-157.

6. Alimian M, Imani F, Faiz SH, et al. Effect of oral pregabalin premedication on post-operative pain in laparoscopic gastric bypass surgery. Anesth Pain Med. 2012;2(1):12-16.

7. Brogi E, Kazan R, Cyr S, et al. Transversus abdominal plane block for postoperative analgesia: a systematic review and meta-analysis of randomized-controlled trials. Can J Anaesth. 2016;63(10):1184-1196.

8. Wassef M, Lee DY, Levine JL, et al. Feasibility and analgesic efficacy of the transversus abdominis plane block after single-port laparoscopy in patients having bariatric surgery. J Pain Res. 2013;6:837-841.

9. Albrecht E, Kirkham KR, Endersby RV, et al. Ultrasound-guided transversus abdominis plane (TAP) block for laparoscopic gastricbypass surgery: a prospective randomized controlled double-blinded trial. Obes Surg. 2013;23(8):1309-1314.

10. Ripollés J, Mezquita SM, Abad A, et al. Analgesic efficacy of the ultrasound-guided blockade of the transversus abdominis plane - a systematic review. Braz J Anesthesiol. 2015;65(4):255-280.

11. Sinha A, Jayaraman L, Punhani D. Efficacy of ultrasound-guided transversus abdominis plane block after laparoscopic bariatric surgery: a double blind, randomized, controlled study. Obes Surg. 2013;23(4):548553.

12. Ruiz-Tovar J, Muñoz JL, Gonzalez J, et al. Postoperative pain after laparoscopic sleeve gastrectomy: comparison of three analgesic schemes (isolated intravenous analgesia, epidural analgesia associated with intravenous analgesia and port-sites infiltration with bupivacaine associated with intravenous analgesia). Surg Endosc. 2017;31(1):231236.

13. Moncada R, Martinaitis L, Landecho M, et al. Does Preincisional Infiltration with Bupivacaine Reduce Postoperative Pain in Laparoscopic Bariatric Surgery? Obes Surg. 2016;26(2):282-288.

14. Symons JL, Kemmeter PR, Davis AT, et al. A double-blinded, prospective randomized controlled trial of intraperitoneal bupivacaine in laparoscopic roux-en-Y gastric bypass. J Am Coll Surg. 2007;204(3):392-398. 
15. Alkhamesi NA, Kane JM, Guske PJ, et al. Intraperitoneal aerosolization of bupivacaine is a safe and effective method in controlling postoperative pain in laparoscopic Roux-en-Ygastric bypass. J Pain Res. 2008;1:9-13.

16. Cottam DR, Fisher B, Atkinson J, et al. A randomized trial of bupivicaine pain pumps to eliminate the need for patient controlled analgesia pumps in primary laparoscopic Roux-en-Y gastric bypass. Obes Surg. 2007;17(5):595-600.

17. Bakhamees HS, El-halafawy YM, Elkerdawy HM, et al. Effects of dexmedetomidine in morbidly obese patients undergoing laparoscopic gastric bypass. Middle East J Anesthesiol. 2007;19(3):537-551.

18. Tufanogullari B, White PF, Peixoto MP, Kianpour D, Lacour T,et al. Dexmedetomidine infusion during laparoscopic bariatric surgery:the effect on recovery outcome variables. Anesth Analg. 2008;106(6):17411748 .

19. Govindarajan R, Ghosh B, Sathyamoorthy MK, et al. Efficacy of ketorolac in lieu of narcotics in the operative management of laparoscopic surgery for morbid obesity. Surg Obes Relat Dis. 2005;1(6):530-535.

20. Hasanein R, El-Sayed W, Nashwa N, et al. The effect of combined remifentanil and low dose ketamine infusion in patients undergoing laparoscopic gastric bypass. Egypt J Anaesthesiol. 2011;27(4):255-260.

21. Song K, Melroy MJ, Whipple OC. Optimizing multimodal analgesia with intravenous acetaminophen and opioids in postoperative bariatric patients. Pharmacotherapy. 2014;34(Suppl 1):14S-21S

22. Lam KK, Mui WL. Multimodal analgesia model to achieve low postoperative opioid requirement following bariatric surgery. Hong Kong Med J. 2016;22(5):428-434.

23. Garimella V, Christina C. Postoperative Pain Control. Clin Colon Rectal Surg. 2013;26(3):191-196.

24. Ahmed A, Latif N, Khan R. Post-operative analgesia for major abdominal surgery and its effectiveness in a tertiary care hospital. Anaesthesiol Clin Pharmacol. 2013;29(4):472-477.
25. Vallano A, Aguilera C, Arnau JM, et al. Management of postoperative pain in abdominal surgery in Spain. A multicentre drug utilization study. Br J Clin Pharmacol. 1999;47(6):667-673.

26. Bjordal JM, Johnson MI, Ljunggreen AE. Transcutaneous electrical nerve stimulation (TENS) can reduce postoperative analgesic consumption. A meta-analysis with assessment of optimal treatment parameters for postoperative pain. Eur J Pain. 2003;7(2):181-188.

27. Zhang J, Ho KY, Wang Y. Efficacy of pregabalin in acute postoperative pain: a meta-analysis. Br J Anaesth. 2011;106(4):454-462.

28. Bhangu A, Singh P, Fitzgerald JE, et al. Postoperative nonsteroidal anti-inflammatory drugs and risk of anastomotic leak: meta-analysis of clinical and experimental studies. World J Surg. 2014;38(9):2247-2257.

29. El-labban GM, Hokkam EN, El-labban MA, et al. Intraincisional vs intraperitoneal infiltration of local anaesthetic for controlling early post-laparoscopic cholecystectomy pain. J Minim Access Surg. 2011;7(3):173-177.

30. Schnabel A, Meyer-Frießem $\mathrm{CH}$, Reichl SU, et al. Is intraoperative dexmedetomidine a new option for postoperative pain treatment? A meta-analysis of randomized controlled trials. Pain. 2013;154(7):1140 1149.

31. McNicol ED, Ferguson MC, Haroutounian S, et al. Single dose intravenous paracetamol or intravenous propacetamol for postoperative pain. Cochrane Database Syst Rev. 2016 ;(5):CD007126.

32. Moon YE, Lee YK, Lee J, et al. The effects of preoperative intravenous acetaminophen in patients undergoing abdominal hysterectomy. Arch Gynecol Obstet. 2011;284(6):1455-1460.

33. De Oliveira GS Jr, Almeida MD, Benzon HT, et al. Perioperative single dose systemic dexamethasone for postoperative pain: a meta-analysis of randomized controlled trials. Anesthesiology. 2011;115(3):575-588.

34. McNicol ED, Ferguson MC, Hudcova J. Patient controlled opioid analgesia versus non-patient controlled opioid analgesia for postoperative pain. Cochrane Database Syst Rev. 2015;6:CD003348. 